\title{
PADUA AND PISA \\ ARE EXPONENTIALLY FAR APART
}

\author{
Benjamin M. M. DE Weger*
}

\begin{abstract}
We answer the question posed by Ian Stewart which Padovan numbers are at the same time Fibonacci numbers. We give a result on the difference between Padovan and Fibonacci numbers, and on the growth of Padovan numbers with negative indices.
\end{abstract}

\section{Introduction}

\subsection{What this paper is not about.}

This paper has nothing to do with Italian topography. It is about Padovan numbers and their distances to Fibonacci numbers. Briefly said the main result of this paper is an explicit lower bound for these distances, which grows exponentially. The problem solved here is a generalization of a question asked by Ian Stewart in his Scientific American Mathematical Recreations column $[\mathbf{S}]$. In this column Stewart described the similarities between Padovan and Fibonacci numbers, and remarked that, appropriately, Pisa, the city of Fibonacci, and Padua, the city with Italian name Padova, are roughly only 100 miles apart. As we show that Padovan and Fibonacci numbers are far apart, this might serve as an explanation for our title.

\subsection{Padovan and Fibonacci numbers.}

The Padovan numbers $P_{m}$, named after Richard Padovan, are defined by

$$
P_{0}=1, \quad P_{1}=0, \quad P_{2}=0, \quad P_{m+1}=P_{m-1}+P_{m-2},
$$

*This author's research was supported by the Netherlands Mathematical Research Foundation SWON with financial aid from the Netherlands Organization for Scientific Research NWO. This paper was written while the author enjoyed the hospitality of the Centre de Recerca Matemàtica, Institut d'Estudis Catalans, Bellaterra, Catalunya. 
and the Fibonacci numbers $F_{n}$, named after Leonardo 'Fibonacci' Pisano, are defined by

$$
F_{0}=0, \quad F_{1}=1, \quad F_{n+1}=F_{n}+F_{n-1} .
$$

Note that in our definition the indices of the Padovan numbers are shifted by 5 compared to Stewart's definition. We list a few of these numbers:

\begin{tabular}{|c|rrrrrrrrrrr|}
\hline$m$ & 0 & 1 & 2 & 3 & 4 & 5 & 6 & 7 & 8 & 9 & 10 \\
\hline$P_{m}$ & 1 & 0 & 0 & 1 & 0 & 1 & 1 & 1 & 2 & 2 & 3 \\
\hline$m$ & 11 & 12 & 13 & 14 & 15 & 16 & 17 & 18 & 19 & 20 \\
\hline$P_{m}$ & 4 & 5 & 7 & 9 & 12 & 16 & 21 & 28 & 37 & 49 \\
\hline$m$ & 21 & 22 & 23 & 24 & 25 & 26 & 27 & 28 & 29 & $\ldots$ \\
\hline$P_{m}$ & 65 & 86 & 114 & 151 & 200 & 265 & 351 & 465 & 616 & $\ldots$ \\
\hline
\end{tabular}

\begin{tabular}{|c|rrrrrrrrrrr|}
\hline$n$ & 0 & 1 & 2 & 3 & 4 & 5 & 6 & 7 & 8 & 9 & 10 \\
\hline$F_{n}$ & 0 & 1 & 1 & 2 & 3 & 5 & 8 & 13 & 21 & 34 & 55 \\
\hline
\end{tabular}

\begin{tabular}{|r|rrrrrrl|}
\hline$n$ & 11 & 12 & 13 & 14 & 15 & 16 & $\ldots$ \\
\hline$F_{n}$ & 89 & 144 & 233 & 377 & 610 & 987 & $\ldots$ \\
\hline
\end{tabular}

It will be clear from the definition that Padovan and Fibonacci numbers can be extended to negative indices. For the Fibonacci numbers this does not give essentially new numbers, as $F_{-n}=(-1)^{n+1} F_{n}$. But the Padovan numbers lack such symmetry, and thus are interesting for negative indices too. We also list a few of them:

\begin{tabular}{|r|rrrrrrrrrr|}
\hline$m$ & 1 & 2 & 3 & 4 & 5 & 6 & 7 & 8 & 9 & 10 \\
\hline$P_{-m}$ & -1 & 1 & 0 & -1 & 2 & -2 & 1 & 1 & -3 & 4 \\
\hline$m$ & 11 & 12 & 13 & 14 & 15 & 16 & 17 & 18 & 19 & 20 \\
\hline$P_{-m}$ & -3 & 0 & 4 & -7 & 7 & -3 & -4 & 11 & -14 & 10 \\
\hline$m$ & 21 & 22 & 23 & 24 & 25 & 26 & 27 & 28 & 29 & 30 \\
\hline$P_{-m}$ & 1 & -15 & 25 & -24 & 9 & 16 & -40 & 49 & -33 & -7 \\
\hline$m$ & 31 & 32 & $\ldots$ & & & & & & & \\
\hline$P_{-m}$ & 56 & -89 & $\ldots$ & & & & & & & \\
\hline
\end{tabular}


These short tables already illustrate the growth behaviour of the sequences. Whereas $P_{m}$ and $F_{n}$ grow very regularly, indeed exponentially, with $F_{n}$ showing the faster growth rate, the $P_{-m}$ show oscillating behaviour, with still exponentially but slowly growing amplitude. These observations are warranted by the following lemma, which is easy to prove, e.g. by mathematical induction.

\section{Lemma 1.}

(i) Let $\gamma$ be the real root of $x^{3}-x-1$, and let $\delta$ be the non-real root of $x^{3}-x-1$ with positive imaginary part. Let

$$
\lambda=\frac{1}{23}\left(5-6 \gamma+4 \gamma^{2}\right), \quad \mu=\frac{1}{23}\left(5-6 \delta+4 \delta^{2}\right) .
$$

Then for all $m \in \mathbb{Z}$

$$
P_{m}=\lambda \gamma^{m}+\mu \delta^{m}+\bar{\mu} \bar{\delta}^{m}
$$

(ii) Let

$$
\alpha=\frac{1}{2}(1+\sqrt{5}), \quad \beta=\frac{1}{2}(1-\sqrt{5}) .
$$

Then for all $n \in \mathbb{Z}$

$$
F_{n}=\frac{1}{\sqrt{5}} \alpha^{n}-\frac{1}{\sqrt{5}} \beta^{n} .
$$

Because $|\beta|<1$ the term $\frac{1}{\sqrt{5}} \beta^{n}$ tends to 0 as $n$ grows, so that this lemma implies at once that $F_{n} \sim \frac{1}{\sqrt{5}} \alpha^{n}$ as $n \rightarrow \infty$, indeed showing exponential growth with growth rate $\alpha=1.61 \ldots$.

Similarly, because $|\delta|<1$, the terms $\mu \delta^{m}$ and $\bar{\mu} \bar{\delta}^{m}$ tend to 0 as $m$ grows, so that the lemma shows that $P_{m} \sim \lambda \gamma^{m}$ as $m \rightarrow \infty$, indeed showing exponential growth with growth rate $\gamma=1.32 \ldots$. Note that $P_{m}$ is the nearest integer to $\lambda \gamma^{m}$ if $m \geq 5$.

When studying $P_{m}$ for negative indices we prefer to write

$$
P_{-m}=\lambda \gamma^{-m}+\mu \delta^{-m}+\bar{\mu} \bar{\delta}^{-m}
$$

with $m$ positive. Now it's the term $\lambda \gamma^{-m}$ that tends to 0 as $m \rightarrow \infty$, whereas the other two terms in the above expression for $P_{-m}$ grow exponentially in absolute value. Notice that

$$
\mu \delta^{-m}+\bar{\mu} \bar{\delta}^{-m}=\mu \delta^{-m}\left(1+\frac{\bar{\mu} \bar{\delta}^{-m}}{\mu \delta^{-m}}\right),
$$


where the number $\frac{\bar{\mu} \bar{\delta}^{-m}}{\mu \delta^{-m}}$ is on the unit circle (and travels around it as $m$ varies). This explains the oscillating behaviour. The amplitude is $\left|\delta^{-m}\right|$, which is equal to $\gamma^{m / 2}$, since $\gamma \delta \bar{\delta}=1$, and thus $|\delta|=(\delta \bar{\delta})^{1 / 2}=\gamma^{-1 / 2}$. Thus the growth rate of the amplitude is only $\gamma^{1 / 2}=1.15 \ldots$.

\subsection{Stewart's first question.}

Stewart in his column $[\mathbf{S}]$ asked two specific questions on Padovan numbers with nonnegative indices. First, he remarks that some Padovan numbers are Fibonacci numbers too, namely

$$
\begin{aligned}
P_{1}=P_{2}=P_{4} & =0=F_{0}, \\
P_{0}=P_{3}=P_{5}=P_{6}=P_{7} & =1=F_{1}=F_{2}, \\
P_{8}=P_{9} & =2=F_{3}, \\
P_{10} & =3=F_{4}, \\
P_{12} & =5=F_{5}, \\
P_{17} & =21=F_{8},
\end{aligned}
$$

and asks whether there are other solutions, and whether the number of solutions is finite or infinite. In this paper we answer this question in showing that there are no other solutions.

Stewart did not care about Padovan numbers with negative indices. Had he done so, he certainly would have noticed (also counting $P_{m}=-F_{n}$ as solution) that

$$
\begin{aligned}
P_{-3}=P_{-12} & =0=F_{0}, \\
-P_{-1}=P_{-2}=-P_{-4}=P_{-7}=P_{-8}=P_{-21} & =1=F_{1}=F_{2}, \\
P_{-5}=-P_{-6} & =2=F_{3}, \\
-P_{-9}=-P_{-11}=-P_{-16} & =3=F_{4}, \\
-P_{-32} & =89=F_{11} .
\end{aligned}
$$

A natural question is whether these are all the solutions of $P_{-m}= \pm F_{n}$. It follows from results of Evertse $[\mathbf{E}]$ and van der Poorten and Schlickewei $[\mathbf{P S}]$ that the number of solutions is finite, and even an explicit upper bound for the number of solutions can be given. We conjecture that the ones mentioned above are all the solutions, but we have no idea how to attack this problem.

It follows from a result of Mignotte [M2] that an equation of the type $u_{m}=v_{n}$ has only finitely many solutions, and can be solved effectively and practically, when $\left\{u_{m}\right\}$ and $\left\{v_{n}\right\}$ both are recurrence sequences with one dominating root, i.e. of the characteristic roots (such as $\gamma, \delta, \bar{\delta}$ for $\left.\left\{P_{m}\right\}\right)$ one is in absolute value strictly larger than the others $(\gamma)$. Indeed, the very first paper in which a diophantine problem was explicitly solved 
by the methods that we use in this paper, was a problem of this type, cf. Baker and Davenport [BD]. It seems that our present result is the first explicit equation of this type involving a second and a third order recurrence sequence.

\subsection{Stewart's second question.}

Stewart's second question is which Padovan numbers are squares. There obviously are the solutions

$$
\begin{aligned}
P_{1}=P_{2}= & P_{4}=0^{2}, \\
P_{0}=P_{3}=P_{5}=P_{6}=P_{7} & =1^{2}, \\
P_{11} & =2^{2}, \\
P_{14} & =3^{2}, \\
P_{20} & =7^{2} .
\end{aligned}
$$

We have no idea how to prove anything in this direction. Stewart might also have noticed that

$$
\begin{array}{r}
P_{-3}=P_{-12}=0^{2}, \\
-P_{-1}=P_{-2}=-P_{-4}=P_{-7}=P_{-8}=P_{-21}=1^{2}, \\
P_{-10}=P_{-13}=-P_{-17}=2^{2}, \\
P_{-25}=3^{2}, \\
P_{-26}=4^{2}, \\
P_{-23}=5^{2}, \\
P_{-28}=7^{2},
\end{array}
$$

but he didn't. Again we have no clue whatsoever how to proceed with this problem.

\subsection{Results I.}

However, we can answer two other questions Stewart did not ask. The first one we get almost for free from our method for solving $P_{m}=F_{n}$. Namely we can prove the following result.

\section{Theorem 2.}

(i) If $m$ and $n$ are nonnegative integers such that $\left|P_{m}-F_{n}\right| \leq P_{m}^{1 / 2}$, then $m \leq 29$ and $n \leq 15$.

(ii) If $m$ and $n$ are nonnegative integers such that $P_{m} \neq F_{n}$ then

$$
\begin{aligned}
& \left|P_{m}-F_{n}\right| \\
& >\max \left\{\frac{P_{m}}{m^{1.4615 \times 10^{15}}}, \frac{P_{m}}{e^{1.7950 \times 10^{9}(\log m)^{2}}}, 0.24174 P_{m}^{1 / 2}\right\} .
\end{aligned}
$$


An answer to Stewart's original question to find the solutions of $P_{m}=F_{n}$ follows immediately from (i), upon inspection of the tables in Section 1.2.

In (i) we could in principle have derived a similar result with the exponent $1 / 2$ replaced by any $\delta<1$. A similar remark holds for (ii), with the constant 0.24174 adjusted accordingly. In (ii), when $m$ is larger than approximately $10^{353605}$, the first term in the max expression is the best one. It shows that asymptotically $\left|P_{m}-F_{n}\right| \gg P_{m}^{1-\epsilon}$ for an arbitrarily small $\epsilon>0$, and thus that Padua and Pisa are indeed exponentially far apart (note that $P_{m} \sim \lambda \gamma^{m}$ ). For $m$ smaller than approximately $1.1549 \times 10^{13}$, the third term in the max expression is the best one.

As an immediate application we now are able to instantaneously solve problems of the type $\left|P_{m}-F_{n}\right| \leq 10^{6}$, say. Namely, (i) tells us that there are no solutions with $P_{m} \geq 10^{12}$, and if $P_{m}<10^{12}$ then, by the fact that $P_{m}$ is the nearest integer to $\lambda \gamma^{m}$ for $m \geq 5$, we immediately have $m \leq 104$. The solutions now are easy to determine.

\subsection{Results II.}

The other problem we'll address in this paper is the growth behaviour of $P_{-m}$. As we saw above this sequence oscillates with exponentially growing amplitude, thus shows complicated behaviour, getting small compared to the amplitude when the number $\frac{\bar{\mu} \bar{\delta}^{-m}}{\mu \delta^{-m}}$ on the unit circle happens to come near to -1 . This happens when $\operatorname{Arg} \mu+m \operatorname{Arg} \delta$ is near to an odd integer times $\frac{1}{2} \pi$. Nevertheless there is a result of Mignotte [M1] that yields a lower bound for $\left|P_{-m}\right|$, and in the following theorem we will make this explicit. This might serve as a meager substitute for our inability to solve $P_{-m}= \pm F_{n}$, but this seems more or less to be at the limit of the available methods.

\section{Theorem 3.}

(i) If $m$ is a nonnegative integer such that $\left|P_{-m}\right| \leq \gamma^{m / 4}$, then $m \leq$ 30 .

(ii) If $m$ is a nonnegative integer not equal to 3 or 12 then

$$
\left|P_{-m}\right|>\max \left\{\frac{\gamma^{m / 2}}{m^{8.4019 \times 10^{15}}}, \frac{\gamma^{m / 2}}{e^{6.3164 \times 10^{11}(\log m)^{2}}}, 0.22848 \gamma^{m / 4}\right\}
$$

From (i) it follows that the sequence $\left\{P_{m}\right\}_{m \in \mathbb{Z}}$ has exactly 5 zeroes, namely at $m=-12,-3,1,2,4$. This result is due to Beukers $[\mathbf{B}]$, with a different proof. 
Similar remarks as those immediately following the statement of Theorem 2 can be made here. Especially we want to remark that asymptotically $\left|P_{-m}\right| \gg \gamma^{(m / 2)(1-\epsilon)}$ for an arbitrarily small $\epsilon>0$, and thus although in the oscillation $P_{-m}$ can become a bit smaller than the amplitude $\gamma^{m / 2}$, this never becomes really dramatic. Also, an application such as finding all solutions to $\left|P_{-m}\right| \leq 10^{6}$ is now instantaneous: from (i) we obtain $\gamma^{m}<10^{24}$, hence $m \leq 196$.

\subsection{Perrin numbers.}

Stewart in his column also considers the Perrin numbers $A_{\ell}$, defined like the Padovan numbers by $A_{\ell+1}=A_{\ell-1}+A_{\ell-2}$, but with different initial conditions: $A_{0}=3, A_{1}=0, A_{2}=2$. They satisfy $A_{\ell}=\gamma^{\ell}+\delta^{\ell}+\bar{\delta}^{\ell}=3 P_{\ell}+2 P_{\ell+1}$. Our methods will certainly be able to prove the following assertions, or in case they are false, to prove similar assertions with the constants replaced by the correct ones. We leave details of such proofs to the interested reader.

\section{Assertions 4.}

(i) If $\ell$ and $n$ are nonnegative integers such that $\left|A_{\ell}-F_{n}\right| \leq A_{\ell}^{1 / 2}$, then $\ell \leq 29$ and $n \leq 18$.

(ii) If $\ell$ and $n$ are nonnegative integers such that $A_{\ell} \neq F_{n}$ then

$$
\left|A_{\ell}-F_{n}\right|>\max \left\{\frac{A_{\ell}}{\ell^{10^{16}}}, \frac{A_{\ell}}{e^{10^{10}(\log \ell)^{2}}}, 0.10540 A_{\ell}^{1 / 2}\right\} .
$$

(iii) If $\ell$ is a nonnegative integer such that $\left|A_{-\ell}\right| \leq \gamma^{\ell / 4}$, then $m \leq 29$.

(iv) If $\ell$ is a nonnegative integer then

$$
\left|A_{-\ell}\right|>\max \left\{\frac{\gamma^{\ell / 2}}{\ell^{10^{16}}}, \frac{\gamma^{\ell / 2}}{e^{10^{12}(\log \ell)^{2}}}, 0.13019 \gamma^{\ell / 4}\right\} .
$$

\section{Proof of Theorem 2}

\subsection{Preparations.}

We start with noting that

$$
\begin{array}{rlrl}
\alpha & =1.61803 \ldots, & \beta & =-0.61803 \ldots, \\
\gamma & =1.32471 \ldots, & \delta & =-0.66235 \ldots+0.56227 \ldots i, \\
\gamma \delta \bar{\delta} & =1, & |\delta| & =\gamma^{-1 / 2}=0.86883 \ldots, \\
\lambda & =0.17700 \ldots, \quad \mu & =0.41149 \ldots-0.27622 \ldots i \\
\lambda \mu \bar{\mu} & =\frac{1}{23}, & |\mu| & =(23 \lambda)^{-1 / 2}=0.49560 \ldots
\end{array}
$$


Lemma 1 gives us at once that

$$
\left|P_{m}-F_{n}\right| \geq\left|\frac{1}{\sqrt{5}} \alpha^{n}-\lambda \gamma^{m}\right|-\left(\frac{1}{\sqrt{5}} \alpha^{-n}+2|\mu| \gamma^{-m / 2}\right)
$$

We may assume without loss of generality that $m \geq 11$, so that $P_{m} \geq 4$. From Lemma 1 we have

$$
\left|P_{m}-\lambda \gamma^{m}\right| \leq 2|\mu| \gamma^{-m / 2} \leq 2|\mu| \gamma^{-11 / 2}<0.21111
$$

so that by $m \geq 11$ and $P_{m} \geq 4$ we find inequalities that we will use repeatedly:

$$
\begin{aligned}
& \text { (5) } \quad \lambda \gamma^{m}>P_{m}-0.21111 \geq\left(1-\frac{0.21111}{4}\right) P_{m}>0.94722 P_{m}, \\
& \text { (6) } \quad P_{m}>\lambda \gamma^{m}-0.21111 \geq\left(1-\frac{0.21111}{\lambda \gamma^{11}}\right) \lambda \gamma^{m}>0.94590 \lambda \gamma^{m} .
\end{aligned}
$$

Next we want to estimate the error term in (4). A rough estimate is

$$
\frac{1}{\sqrt{5}} \alpha^{-n}+2|\mu| \gamma^{-m / 2}<\frac{1}{\sqrt{5}}+2|\mu| \gamma^{-11 / 2}<0.65832
$$

But we can do much better. First we remark that if in some cases we can prove that $\left|P_{m}-F_{n}\right|>c \lambda \gamma^{m}$ for some constant $c>0$, then we are essentially done, as it immediately follows that $\left|P_{m}-F_{n}\right|>c^{\prime} P_{m}$ for some other constant $c^{\prime}>0$. In the case that

$$
\alpha^{-n} \geq \gamma^{-m / 2}
$$

we have

$$
\frac{1}{\sqrt{5}} \alpha^{n}-\lambda \gamma^{m} \leq \frac{1}{\sqrt{5}} \gamma^{m / 2}-\lambda \gamma^{m}<0,
$$

so by (4) and (7)

$$
\begin{aligned}
\left|P_{m}-F_{n}\right| & \geq \lambda \gamma^{m}-\frac{1}{\sqrt{5}} \alpha^{n}-0.65832 \geq \lambda \gamma^{m}\left(1-\frac{1}{\lambda \gamma^{m / 2} \sqrt{5}}\right)-0.65832 \\
& \quad \geq \lambda \gamma^{m}\left(1-\frac{1}{\lambda \gamma^{11 / 2} \sqrt{5}}-\frac{0.65832}{\lambda \gamma^{11}}\right) \\
& >0.29323 \lambda \gamma^{m}
\end{aligned}
$$


and with (5) this yields

$$
\left|P_{m}-F_{n}\right|>0.27775 P_{m} .
$$

So in this case $P_{m} \neq F_{n}$, and $\left|P_{m}-F_{n}\right| \leq P_{m}^{1 / 2}$ at once implies $P_{m} \leq 12$, and also the inequality (2) is obvious in this case.

So we may assume that

$$
\alpha^{-n}<\gamma^{-m / 2}
$$

and then we find for the error term in (4) a much better estimate than (7), namely

(9) $\frac{1}{\sqrt{5}} \alpha^{-n}+2|\mu| \gamma^{-m / 2}<\left(\frac{1}{\sqrt{5}}+2|\mu|\right) \gamma^{-m / 2}<1.4385 \gamma^{-m / 2}$.

To deal with the main term of (4) we introduce a linear form in logarithms of algebraic numbers:

$$
\Lambda=-\log (\lambda \sqrt{5})+n \log \alpha-m \log \gamma .
$$

Notice that

$$
\left|\frac{1}{\sqrt{5}} \alpha^{n}-\lambda \gamma^{m}\right|=\lambda \gamma^{m}\left|e^{\Lambda}-1\right| .
$$

It follows that $\Lambda \neq 0$. In the case $\Lambda \leq-1$ we have $\left|e^{\Lambda}-1\right|=1-e^{\Lambda} \geq$ $1-e^{-1}$, so by $(4),(7)$ and (10) we find

$$
\begin{aligned}
& \left|P_{m}-F_{n}\right| \geq \lambda \gamma^{m}\left(1-e^{-1}\right)-0.65832 \\
& \quad \geq \lambda \gamma^{m}\left(1-e^{-1}-\frac{0.65832}{\lambda \gamma^{11}}\right)>0.46343 \lambda \gamma^{m},
\end{aligned}
$$

which is covered by (8). So we may assume that $\Lambda>-1$. Then $\left|e^{\Lambda}-1\right|>\left(1-e^{-1}\right)|\Lambda|$, and with (10) this gives us an important estimate:

$$
\left|\frac{1}{\sqrt{5}} \alpha^{n}-\lambda \gamma^{m}\right|>0.63212 \lambda \gamma^{m}|\Lambda| .
$$

The last special case we have to deal with is the case $n>m$. Then $n \geq m+1 \geq 12$, so

$$
\Lambda \geq \log \frac{\alpha}{\lambda \sqrt{5}}+11 \log \frac{\alpha}{\gamma}>3.6081 .
$$

With (4), (7) and (12) this yields

(13) $\left|P_{m}-F_{n}\right|>2.2807 \lambda \gamma^{m}-0.65832$

$$
\geq \lambda \gamma^{m}\left(2.2807-\frac{0.65832}{\lambda \gamma^{11}}\right)>2.1120 \lambda \gamma^{m},
$$

which again is covered by (8). So we may assume that $n \leq m$. 


\subsection{Application of transcendence theory.}

Now we are ready to apply the main ingredients: explicit lower bounds for $|\Lambda|$, that transcendence theory provides. We use the results of Baker and Wüstholz $[\mathbf{B W}]$, which give the asymptotically best results, and of Voutier $[\mathbf{V}]$, which gives the best results for small $m$. Notice that our linear form has three terms, and the field $\mathbb{Q}(\alpha, \gamma)$ is of degree 6. By $\Lambda \neq 0$ the theorem of $[\mathbf{B W}]$ and Theorem 3 of $[\mathbf{V}]$ give

$$
|\Lambda|>\max \left\{m^{-C_{B W}}, e^{-C_{V}(\log m)^{2}}\right\},
$$

where $C_{B W}$ and $C_{V}$ are large absolute constants that we can compute explicitly.

Indeed, for $C_{B W}$ we have

$$
C_{B W}=18 \times 4 ! \times 3^{4} \times 192^{5} \times \log 36 \times h^{\prime}(\lambda \sqrt{5}) \times h^{\prime}(\alpha) \times h^{\prime}(\gamma) .
$$

Here the function $h^{\prime}$ on our sextic field is defined by

$$
h^{\prime}(\xi)=\max \left\{h(\xi), \frac{1}{6}|\log \xi|, \frac{1}{6}\right\},
$$

where $h$ is the absolute logarithmic Weil height defined by

$$
h(\xi)=\frac{1}{d}\left(\log a_{0}+\sum_{\sigma} \max \{0, \log |\sigma(\xi)|\}\right),
$$

where the sum runs over all $d$ embeddings $\sigma$ of the field $\mathbb{Q}(\xi)$ into $\mathbb{C}, d$ is the degree of this field, and $a_{0}$ is the leading coefficient of the minimal polynomial of $\xi$. It is easy to compute the required values for $h^{\prime}$ (on noting that for $\lambda \sqrt{5}$ we have $a_{0}=23^{2}$ ), and we find

$$
h^{\prime}(\lambda \sqrt{5})<1.1137, \quad h^{\prime}(\alpha)<0.24061, \quad h^{\prime}(\gamma)=\frac{1}{6} .
$$

So for the constant $C_{B W}$ in (14) this leads to

$$
C_{B W}<1.4613 \times 10^{15} \text {. }
$$

And we have

$$
C_{V}=285000 \times 6^{5} \times 4 \times h^{\prime \prime}(\lambda \sqrt{5}) \times h^{\prime \prime}(\alpha) \times h^{\prime \prime}(\gamma),
$$

where the function $h^{\prime \prime}$ on our sextic field is defined by

$$
h^{\prime \prime}(\xi)=\max \left\{h(\xi), \frac{3.4}{3}|\log \xi|, \frac{1}{3}\right\} .
$$


We find

$$
h^{\prime \prime}(\lambda \sqrt{5})<1.1137, \quad h^{\prime \prime}(\alpha)<0.54538, \quad h^{\prime \prime}(\gamma)=\frac{1}{3} .
$$

Notice that in applying Voutier's Theorem 3 we have taken $B=m^{2}$ (whence the factor 4 ), which by $n \leq m$ is larger than

$$
\left(\frac{1}{3 h^{\prime \prime}(\gamma)}+\frac{m}{3 h^{\prime \prime}(\lambda \sqrt{5})}\right)\left(\frac{n}{3 h^{\prime \prime}(\gamma)}+\frac{m}{3 h^{\prime \prime}(\alpha)}\right)
$$

For the constant $C_{V}$ in (14) this leads to

$$
C_{V}<1.7948 \times 10^{9}
$$

\subsection{Finishing the proof.}

Now we combine (4), (9), (12), (14) and (16), to obtain

$$
\left|P_{m}-F_{n}\right|>0.63212 \frac{\lambda \gamma^{m}}{e^{1.7948 \times 10^{9}(\log m)^{2}}}-1.4385 \gamma^{-m / 2} .
$$

Note that e.g. when $m=17$, where there is a solution of $P_{m}=F_{n}$, the right hand side of (17) is negative, so the inequality is still true, but useless. Of course (17) is useful only if the right hand side is positive, and that happens if $m \geq 3.5537 \times 10^{12}$. Thus at this point we have proved that if $P_{m}=F_{n}$ then $m<3.5537 \times 10^{12}$. That's at least something.

If $m \geq 3.5537 \times 10^{12}$ then

$$
\frac{0.63212}{1.4385} \frac{\lambda \gamma^{\frac{3}{2} m}}{e^{1.7948 \times 10^{9}(\log m)^{2}}}>e^{1.6138 \times 10^{7}} .
$$

It follows from (17) that

$$
\left|P_{m}-F_{n}\right|>0.63212 \frac{\lambda \gamma^{m}}{e^{1.7948 \times 10^{9}(\log m)^{2}}}\left(1-e^{-1.61380 \times 10^{7}}\right) .
$$

Finally it follows by (5) that

$(18) \quad$ if $\quad m \geq 3.5537 \times 10^{12} \quad$ then $\quad\left|P_{m}-F_{n}\right|>\frac{P_{m}}{e^{1.7949 \times 10^{9}(\log m)^{2}}}$,

which is a major step towards the proof of (ii).

Assume that

$$
\left|P_{m}-F_{n}\right| \leq P_{m}^{1 / 2}
$$


Then (18) implies, if $m \geq 3.5537 \times 10^{12}$, that

$$
\frac{P_{m}}{e^{1.7949 \times 10^{9}}(\log m)^{2}}<P_{m}^{1 / 2},
$$

and thus, by (6),

$$
0.94590 \lambda \gamma^{m}<P_{m}<e^{3.5898 \times 10^{9}(\log m)^{2}},
$$

and this implies $m<1.1549 \times 10^{13}$. Thus we have

(19) if $m \geq 1.1549 \times 10^{13}$ then $\left|P_{m}-F_{n}\right|>P_{m}^{1 / 2}>0.24174 P_{m}^{1 / 2}$,

which is another major step towards the proof of both (i) and (ii).

In the next subsection we will show that if $m<1.1549 \times 10^{13}$ then $\left|P_{m}-F_{n}\right| \leq P_{m}^{1 / 2}$ has only the solutions mentioned in the statement (i) in the theorem. Assuming this for the moment, we can now finish the proof of (ii). Namely, from inspection of the solutions of $\left|P_{m}-F_{n}\right| \leq P_{m}^{1 / 2}$ we find that there are no solutions with $0<\left|P_{m}-F_{n}\right| \leq 0.24174 P_{m}^{1 / 2}$. Indeed, the smallest nonzero value of $\left|P_{m}-F_{n}\right| / P_{m}^{1 / 2}$ occurs for $P_{29}=616$ and $F_{15}=610$, which explains the number $0.24174<0.241746 \ldots=\frac{6}{\sqrt{616}}$. This proves that

$$
\text { if } m<1.1549 \times 10^{13} \text { then }\left|P_{m}-F_{n}\right|>0.24174 P_{m}^{1 / 2} \text {. }
$$

Further, by $m<1.1549 \times 10^{13}$ it follows that

$$
\sqrt{\lambda} \gamma^{m / 2}<0.23527 e^{1.7950 \times 10^{9}(\log m)^{2}},
$$

which by (5) implies that

$$
P_{m}^{1 / 2}<0.24174 e^{1.7950 \times 10^{9}(\log m)^{2}}
$$

and thus by (20) we have

(21) if $m<1.1549 \times 10^{13}$

$$
\text { then }\left|P_{m}-F_{n}\right|>0.24174 P_{m}^{1 / 2}>\frac{P_{m}}{e^{1.7950 \times 10^{9}(\log m)^{2}}} .
$$

Now (18), (19), (20) and (21) together imply two of the three bounds of (ii), and the third one follows by a similar reasoning, of which we do not give the details. 


\subsection{Application of computational diophantine approxima-} tion.

It remains to prove that if $m<1.1549 \times 10^{13}$ then the inequality $\left|P_{m}-F_{n}\right| \leq P_{m}^{1 / 2}$ has no solutions with $m \geq 30$ (notice that the solutions with $m \leq 29$ are very easy to find). This we do by a computational diophantine approximation technique, known as the Baker-Davenport method. Here the linear form $\Lambda$ will play a crucial rôle. See $[\mathbf{d W}]$ for a more complete description of these techniques.

From (12), (4) and (7) we find

$$
|\Lambda|<\frac{1}{0.63212 \lambda \gamma^{m}}\left(\left|P_{m}-F_{n}\right|+1.4385 \gamma^{-m / 2}\right),
$$

and thus using our inequality and (5) and $m \geq 30$ we have

$$
|\Lambda|<\frac{1}{0.63212 \lambda \gamma^{m}}\left(\frac{1}{\sqrt{0.94722}} \lambda^{1 / 2}+1.4385 \gamma^{-30}\right) \gamma^{m / 2},
$$

thus

$$
|\Lambda|<3.8663 \gamma^{-m / 2}
$$

Consider the lattice $\Gamma=\left\{\mathcal{C} \mathbf{x} \mid \mathbf{x} \in \mathbb{Z}^{2}\right\}$ defined by the matrix

$$
\mathcal{C}=\left(\begin{array}{cc}
1 & 0 \\
{\left[10^{30} \log \gamma\right.} & {\left[10^{30} \log \alpha\right]}
\end{array}\right)
$$

where $[\cdot]$ stands for rounding to the nearest integer. For $\mathcal{C}$ we computed

$$
\left(\begin{array}{rr}
1 & 0 \\
281199574322961846512050764068 & 481211825059603447497758913424
\end{array}\right) .
$$

So the columns of the matrix $\mathcal{C}$ form a basis of the lattice. Further, consider the point

$$
\mathbf{y}=\left(\begin{array}{c}
0 \\
{\left[10^{30} \log (\lambda \sqrt{5})\right]}
\end{array}\right)=\left(\begin{array}{r}
0 \\
-926836745813687467402313447920
\end{array}\right) .
$$

For a possible solution $(m, n)$ we now look at the distance $d$ between the lattice point $\mathcal{C}\left(\begin{array}{c}-m \\ n\end{array}\right)$ and the point $\mathbf{y}$. This distance is the length of the vector

$$
\mathcal{C}\left(\begin{array}{c}
-m \\
n
\end{array}\right)-\mathbf{y}=\left(\begin{array}{c}
-m \\
\Lambda^{\prime}
\end{array}\right)
$$


with

$$
\Lambda^{\prime}=-\left[10^{30} \log (\lambda \sqrt{5})\right]+n\left[10^{30} \log \alpha\right]-m\left[10^{30} \log \gamma\right] .
$$

The first coordinate of this vector is bounded by our assumption that $m<1.1549 \times 10^{13}$, and the second coordinate is approximately $10^{30} \Lambda$, which is bounded by (22). On the other hand, since the lattice is discrete, the lattice point nearest to $\mathbf{y}$ lies at some distance, that can be computed explicitly.

In order to do this we introduce the concept of a reduced basis of a lattice. That is a basis $\left\{\mathbf{b}_{1}, \mathbf{b}_{2}\right\}$ with the following properties: $\left|\mathbf{b}_{1}\right| \leq\left|\mathbf{b}_{2}\right|$, and $\left|\mathbf{b}_{2}\right| \leq\left|\mathbf{b}_{2}+k \mathbf{b}_{1}\right|$ for all $k \in \mathbb{Z}$. From a given arbitrary basis a reduced basis can easily be computed by a variant of the Euclidean algorithm.

A reduced basis enables us to efficiently decide which lattice point is the nearest to $\mathbf{y}$. Namely, we compute the coordinates of $\mathbf{y}$ with respect to the reduced basis, and round them upwards and downwards to the nearest integers. The four coordinate vectors we thus obtain give rise to four lattice points, one of which will be the nearest, due to the basic properties of the reduced basis. The interested reader is invited to write out the proof of this fact.

A reduced basis of our lattice $\Gamma$ is given by the columns of the matrix

$$
\mathcal{B}=\left(\begin{array}{rr}
-274801712356211 & -676804792431148 \\
-447145768517436 & 649855579430336
\end{array}\right)
$$

Notice that $\mathcal{C}^{-1} \mathcal{B}$ is a unimodular matrix with integral entries, so that the columns of $\mathcal{B}$ indeed constitute a basis for $\Gamma$. For the coordinates of y we find

$$
\mathcal{B}^{-1} \mathbf{y}=\left(\begin{array}{c}
1303558056351372.64600 \ldots \\
-529281101503922.13406 \ldots
\end{array}\right)
$$

Actually, the lattice point nearest to $\mathbf{y}$ indeed is

$\mathcal{B}\left(\begin{array}{r}1303558056351373 \\ -529281101503922\end{array}\right)=\left(\begin{array}{r}-188016117965247 \\ -926836745813687538563322817420\end{array}\right)$,

and its distance to $\mathbf{y}$ is larger than $2.0103 \times 10^{14}$.

It follows by the definition of $d$ and by our upper bound for $m$ that

$$
\left(2.0103 \times 10^{14}\right)^{2} \leq d^{2}=m^{2}+\Lambda^{\prime 2} \leq\left(1.1549 \times 10^{13}\right)^{2}+\Lambda^{\prime 2},
$$

hence

$$
\left|\Lambda^{\prime}\right|>2.0069 \times 10^{14}
$$


We have by the definition of $\Lambda^{\prime}$ and the upper bound for $m$ that

$$
\left|\Lambda^{\prime}-10^{30} \Lambda\right| \leq 1+m+n \leq 1+2 m<2.3099 \times 10^{13},
$$

and so we find

$$
|\Lambda|>1.7759 \times 10^{-16},
$$

and now together with (22) we immediately find that

$$
m \leq 267 \text {. }
$$

We can repeat the game, with $10^{30}$ replaced by $10^{7}$. Now we have

$$
\begin{aligned}
\mathcal{C} & =\left(\begin{array}{cc}
1 & 0 \\
{\left[10^{7} \log \gamma\right.} & {\left[10^{7} \log \alpha\right.}
\end{array}\right)=\left(\begin{array}{rr}
1 & 0 \\
2811995 & 4812118
\end{array}\right), \\
\mathbf{y} & =\left(\begin{array}{cr}
0 & 0 \\
{\left[10^{7} \log (\lambda \sqrt{5})\right.}
\end{array}\right)=\left(\begin{array}{r}
-9268367
\end{array}\right) \\
\mathcal{B} & =\left(\begin{array}{rr}
1547 & 895 \\
1593 & -2189
\end{array}\right) \\
\mathcal{B}^{-1} \mathbf{y} & =\left(\begin{array}{l}
-1723.81235 \ldots \\
-2979.59521 \ldots
\end{array}\right)
\end{aligned}
$$

and again the lattice point nearest to $\mathbf{y}$ is

$$
\mathcal{B}\left(\begin{array}{r}
-1724 \\
2980
\end{array}\right)=\left(\begin{array}{r}
72 \\
-9269552
\end{array}\right)
$$

and its distance to $\mathbf{y}$ is larger than 1187.1. So

$$
1187.1^{2} \leq d^{2}=m^{2}+\Lambda^{\prime 2} \leq 267^{2}+\Lambda^{\prime 2},
$$

hence

$$
\left|\Lambda^{\prime}\right|>1156.6
$$

Also

$$
\left|\Lambda^{\prime}-10^{7} \Lambda\right| \leq 1+m+n \leq 1+2 m \leq 535,
$$

and so we find

$$
|\Lambda|>6.2160 \times 10^{-5}
$$

and now together with $(22)$ we immediately find that

$$
m \leq 78 \text {. }
$$

Finally, finding the solutions with $30 \leq m \leq 78$ can simply be done by enumeration. This completes the proof of Theorem 2 . 


\section{Proof of Theorem 3}

\subsection{Preparations.}

This proof follows to a large extent the line of argument set out in the previous proof ${ }^{1}$, and is in details a bit simpler. For $m \geq 0$ we write (cf. (1))

$$
P_{-m}=\mu \delta^{-m}\left(1+\frac{\bar{\mu}}{\mu}\left(\frac{\delta}{\bar{\delta}}\right)^{m}\right)+\lambda \gamma^{-m},
$$

and notice that $\lambda \gamma^{-m}$ now is exponentially small, that $\left|\mu \delta^{-m}\right|=|\mu| \gamma^{m / 2}$ is large, and that $\frac{\bar{\mu}}{\mu}\left(\frac{\delta}{\bar{\delta}}\right)^{m}$ lies on the unit circle. So we put

$$
\frac{\bar{\mu}}{\mu}=e^{i \psi}, \quad \frac{\delta}{\bar{\delta}}=e^{i \phi},
$$

where we take $\psi, \phi \in(-\pi, \pi]$ (in the sequel, $\pi=3.14159 \ldots$ is the number satisfying $\left.e^{i \pi}=-1\right)$. Indeed,

$$
\psi=1.18235 \ldots, \quad \phi=-1.40771 \ldots .
$$

Without loss of generality we may assume that $m \geq 10$, say. We put

$$
\Lambda=\psi+m \phi+(2 \ell-1) \pi,
$$

where we take $\ell \in \mathbb{Z}$ such that $\Lambda \in(-\pi, \pi]$. Notice that by $m \geq 10$ we have

$$
2 \ell-1=\frac{1}{\pi}(\Lambda-\psi+m|\phi|)<0.44810 m+0.62365<m,
$$

and also $2 \ell-1 \geq 5$. Hence

$$
\max \{|2 \ell-1|, m\}=m .
$$

So we now have

$$
P_{-m}=\mu \delta^{-m}\left(1-e^{i \Lambda}\right)+\lambda \gamma^{-m} .
$$

If $\Lambda \geq \frac{\pi}{3}$ then $\left|e^{i \Lambda}-1\right|=2\left|\sin \left(\frac{1}{2} \Lambda\right)\right| \geq 1$, hence by (24) we have

$$
\left|P_{-m}\right| \geq|\mu| \gamma^{m / 2}-\lambda \gamma^{-m} \geq\left(|\mu|-\lambda \gamma^{-15}\right) \gamma^{m / 2}>0.49300 \gamma^{m / 2} .
$$

${ }^{1}$ As my colleague Henk Hoogland would say: this is going to be a proof by texteditor. The reader interested in writing out a proof of the Assertions 4 can obtain the $\mathrm{LAT}_{\mathrm{E}} \mathrm{X}$-code of this paper from the author upon request. 
In this case the statements of the theorem are immediate.

If $\Lambda<\frac{\pi}{3}$ then $\left|e^{i \Lambda}-1\right|=2\left|\sin \left(\frac{1}{2} \Lambda\right)\right| \geq \frac{3}{\pi}|\Lambda|$, hence (24) gives

$$
\left|P_{-m}\right| \geq|\mu| \gamma^{m / 2} \frac{3}{\pi}|\Lambda|-\lambda \gamma^{-m} .
$$

\subsection{Application of transcendence theory.}

A lower bound for $|\lambda|$ is again furnished by transcendence theory. Indeed, $[\mathbf{B W}]$ and $[\mathbf{V}]$ give (by (23) and on noting that $\Lambda \neq 0$ )

$$
|\Lambda|>\max \left\{m^{-C_{B W}}, e^{-C_{V}(\log m)^{2}}\right\},
$$

where $C_{B W}$ and $C_{V}$ are large absolute constants that can be computed explicitly.

In fact, for $C_{B W}$ we find

$$
C=18 \times 4 ! \times 3^{4} \times 192^{5} \times \log 36 \times h^{\prime}\left(\frac{\bar{\mu}}{\mu}\right) \times h^{\prime}\left(\frac{\delta}{\bar{\delta}}\right) \times h^{\prime}(-1),
$$

where -1 is to be interpreted as $e^{i \pi}$. Now we have the function $h^{\prime}$ on the sextic field $\mathbb{Q}(\delta, \bar{\delta})$. Using $h\left(\frac{\xi}{\bar{\xi}}\right) \leq 2 h(\xi)$ we find

$$
h^{\prime}\left(\frac{\bar{\mu}}{\mu}\right)<2.0904, \quad h^{\prime}\left(\begin{array}{c}
\delta \\
\bar{\delta}
\end{array}\right)<0.23462, \quad h^{\prime}(-1)=\frac{\pi}{6}<0.52360,
$$

so we find for $C_{B W}$ in (26) that

$$
C<8.4017 \times 10^{15} .
$$

And for $C_{V}$ we have

$$
C_{V}=285000 \times 6^{5} \times 4 \times h^{\prime \prime}\left(\frac{\bar{\mu}}{\mu}\right) \times h^{\prime \prime}\left(\frac{\delta}{\bar{\delta}}\right) \times h^{\prime \prime}(-1),
$$

and we find

$$
h^{\prime \prime}\left(\frac{\bar{\mu}}{\mu}\right)<2.0904, \quad h^{\prime \prime}\left(\begin{array}{c}
\delta \\
\bar{\delta}
\end{array}\right)<1.5955, \quad h^{\prime \prime}(-1)=\frac{3.4 \pi}{3}<3.5605 .
$$

Notice that in applying Voutier's Theorem 3 we have taken $B=m^{2}$ (whence the factor 4 ), which by $2 \ell-1 \leq m$ is larger than

$$
\left(\frac{1}{3 h^{\prime \prime}(-1)}+\frac{2 \ell-1}{3 h^{\prime \prime}(\bar{\mu} / \mu)}\right)\left(\frac{m}{3 h^{\prime \prime}(-1)}+\frac{2 \ell-1}{3 h^{\prime \prime}(\delta / \bar{\delta})}\right) \text {. }
$$


For the constant $C_{V}$ in (26) this leads to

$$
C_{V}<6.3162 \times 10^{11} .
$$

\subsection{Finishing the proof.}

Now from (25), (26) and (28) we obtain

$$
\left|P_{-m}\right| \geq 0.47327 \frac{\gamma^{m / 2}}{e^{6.3162 \times 10^{11}(\log m)^{2}}}-\lambda \gamma^{-m} .
$$

The right hand side is positive if $m \geq 1.8506 \times 10^{15}$. So we now have proved that if $P_{-m}=0$ then $m<1.8506 \times 10^{15}$.

If $m \geq 1.8506 \times 10^{15}$ then

$$
\frac{0.47327}{\lambda} \frac{\lambda \gamma^{\frac{3}{2} m}}{e^{6.3162 \times 10^{11}(\log m)^{2}}}>e^{1.0840 \times 10^{10}} .
$$

It then follows from (29) that

$$
\text { (30) if } m \geq 1.8506 \times 10^{15} \text { then }\left|P_{-m}\right|>\frac{\gamma^{m / 2}}{e^{6.3163 \times 10^{11}(\log m)^{2}}} \text {, }
$$

which is a major step towards the proof of (ii).

Assume that

$$
\left|P_{-m}\right| \leq \gamma^{m / 4} .
$$

Then (30) immediately implies that $m<1.2335 \times 10^{16}$. Thus we have

(31) if $m \geq 1.2335 \times 10^{16}$ then $\left|P_{-m}\right|>\gamma^{m / 4}>0.22848 \gamma^{m / 4}$,

which is another major step towards the proof of both (i) and (ii).

In a moment we will show that if $m<1.2335 \times 10^{16}$ then $\left|P_{-m}\right| \leq \gamma^{m / 4}$ has only the solutions mentioned in the statement (i) in the theorem. Assuming this for the moment, we can now finish the proof of (ii) in a similar way as we did in the proof of Theorem 2. Notice that the number $0.22848<0.228482 \ldots=\gamma^{-21 / 4}$ is the smallest nonzero value of $\left|P_{-m}\right| / \gamma^{m / 4}$, that occurs for $P_{-21}=1$. This proves that

$$
\text { if } m<1.2335 \times 10^{16} \text { then }\left|P_{-m}\right|>0.22848 \gamma^{m / 4} .
$$

Further, by $m<1.2335 \times 10^{16}$ it follows that

$$
\gamma^{m / 4}<0.22848 m^{8.4019 \times 10^{15}},
$$

and thus by (32) we have

(33) if $m<1.2335 \times 10^{16}$

$$
\text { then }\left|P_{-m}\right|>0.22848 \gamma^{m / 4}>\frac{\gamma^{m / 2}}{e^{6.3164 \times 10^{15}}} .
$$


Now (30), (31), (32) and (33) together imply two of the three bounds in (ii), and the third one follows by a similar reasoning, of which we do not give the details.

\subsection{Application of computational diophantine approxima-} tion.

It remains to prove that if $m<1.2335 \times 10^{16}$ then the inequality $\left|P_{-m}\right| \leq \gamma^{m / 4}$ has no solutions with $m \geq 31$ (notice that the solutions with $m \leq 30$ are very easy to find). This we do by the same computational diophantine approximation technique that we used in the proof of Theorem 2, using again the linear form $\Lambda$. This time we omit some numerical details.

From (25) and our inequality and $m \geq 31$ we have

$$
|\Lambda| \leq \frac{\pi}{3|\mu|}\left(1+\frac{\lambda}{\gamma^{155 / 4}}\right) \gamma^{-m / 4}
$$

thus

$$
|\Lambda|<2.1130 \gamma^{-m / 4}
$$

Consider the lattice $\Gamma=\left\{\mathcal{C} \mathbf{x} \mid \mathbf{x} \in \mathbb{Z}^{2}\right\}$ defined by the matrix

$$
\mathcal{C}=\left(\begin{array}{cc}
1 & 0 \\
{\left[10^{34} \phi\right]} & {\left[10^{34} \pi\right]}
\end{array}\right),
$$

where [.] stands for rounding to the nearest integer. Further, consider the point

$$
\mathbf{y}=\left(\begin{array}{c}
0 \\
-\left[10^{34} \psi\right]
\end{array}\right)
$$

For a possible solution $m$ the distance $d$ to look at now is the distance between the lattice point $\mathcal{C}\left(\begin{array}{c}m \\ 2 \ell-1\end{array}\right)$ and the point $\mathbf{y}$, being the length of the vector

$$
\mathcal{C}\left(\begin{array}{c}
m \\
2 \ell-1
\end{array}\right)-\mathbf{y}=\left(\begin{array}{c}
m \\
\Lambda^{\prime}
\end{array}\right),
$$

with

$$
\Lambda^{\prime}=-\left[10^{34} \psi\right]+m\left[10^{34} \phi\right]+(2 \ell-1)\left[10^{34} \pi\right] .
$$

The distance $d$ between $\mathbf{y}$ and the nearest lattice point is larger than $8.9716 \times 10^{16}$ (we omit numerical details behind this fact). It follows as in Section 2.4 that

$$
|\Lambda|>6.4192 \times 10^{-18}
$$


and now together with (34) we immediately find that

$$
m \leq 573 .
$$

Again we repeat the game, with $10^{34}$ replaced by $10^{7}$. Now we have $d>2377.3$, and we derive

$$
|\Lambda|>1.1602 \times 10^{-4}
$$

and now together with (34) we immediately find that

$$
m \leq 139 .
$$

Finally, finding the solutions with $31 \leq m \leq 139$ can simply be done by enumeration. This completes the proof of Theorem 3 .

\section{References}

[BD] A. BAKer And H. DAvenport, The equations $3 x^{2}-2=y^{2}$ and $8 x^{2}-7=z^{2}$, Quart. J. Math. Oxford Ser. (2) 20 (1969), 129-137.

[BW] A. BAKer and G. WÜstholz, Logarithmic forms and group varieties, J. Reine Angew. Math. 442 (1993), 19-62.

[B] F. Beukens, The zero-multiplicity of ternary recurrences, Compositio Math. 77 (1991), 165-177.

[E] J.-H. Evertse, On sums of $S$-units and linear recurrences, Compositio Math. 53 (1984), 225-244.

[M1] M. Mignotte, A note on linear recursive sequences, J. Austral. Math. Soc. Ser. A 20 (1975), 241-244.

[M2] M. Mignotte, Une extension du théorème de Skolem-Mahler, C.R. hebd. Séanc. Acad. Sci. Paris Sér. A 288 (1979), 233-235.

[PS] A. J. van Der Poorten and H. P. Schlickewei, Additive relations in fields, J. Austral. Math. Soc. Ser. A 51 (1991), 154-170.

[S] I. Stewart, Mathematical Recreations: Tales of a neglected number, Scientific American 274 (1996), 92-93.

[V] P. M. Voutier, Linear forms in three logarithms, to appear. 
[dW] B. M. M. DE WEgER, Algorithms for diophantine equations, CWI-tract no. 65, Centrum voor Wiskunde en Informatica, Amsterdam (1989).

\author{
Mathematical Institute \\ University of Leiden \\ and \\ Econometric Institute \\ Erasmus University Rotterdam \\ P.O. Box 1738 \\ 3000 DR Rotterdam \\ THE NETHERLANDS \\ e-mail: deweger@few.eur.nl
}

Primera versió rebuda el 14 d'Octubre de 1996 , darrera versió rebuda el 17 de Març de 1997 